\title{
Diversification of Floral Homeotic Gene Function
}

\author{
Vivian F. Irish \\ Departments of Molecular, Cellular, and Developmental Biology and Ecology and Evolutionary Biology \\ Yale University, PO. Box 208104, New Haven, CT 06520
}

\begin{abstract}
Understanding and controlling how flowers develop is of central importance in devising efficient means to regulate both reproduction and floral form. This has both a practical and aesthetic consequence; manipulating reproduction can have a significant impact on the yield of agronomically important species, while altering flower structure is of wide interest to breeders interested in developing new ornamental varieties. Molecular genetic research using Arabidopsis thaliana (mouse ear cress) has greatly facilitated our understanding of the genes and genetic hierarchies controlling various aspects of floral patterning. One component of floral patterning that has been particularly well studied is the specification of different floral organ identities. By manipulating the expression and/or function of the genes regulating this process, Arabidopsis flowers that are male or female sterile, or have unusual arrangements of organ types can be generated. The explosion of information about the genes controlling floral organ identity specification in Arabidopsis has led to the cloning of homologous genes from a variety of other plant species, with the aim of applying the lessons learned in Arabidopsis to species of horticultural and agronomic importance. To what extent, though, are the genetic mechanisms that have been elucidated in Arabidopsis conserved among species with widely varying floral forms? I will review the current research on the specification of floral organ identity in Arabidopsis, with particular emphasis on the genes controlling petal and stamen development, and discuss the ramifications of the idea that changes in the developmental programs controlled by these genes have paralleled the evolution of floral form.
\end{abstract}

\section{Floral patterning in Arabidopsis}

Arabidopsis flowers, like those of other members of the Brassicaceae, consist of four whorls of organ types: four sepals in whorl one, four cruciform petals in whorl two, six stamens (four long and two short) in whorl three and two fused carpels that form the gynoecium in whorl four. In Arabidopsis, a number of floral homeotic mutations have been recovered which disrupt this pattern, and correspond to key genes required for the specification of the different floral organ identities.

Ten years ago, based on genetic analyses of these floral homeotic mutations, Elliot Meyerowitz and Enrico Coen proposed what has come to be known as the ABC model of floral organ identity (Coen and Meyerowitz, 1991). This model postulates three activities, A, B, and $\mathrm{C}$, that act in combination to specify the different organ types. The A function is required in whorls one and two, B in whorls two and three, and $\mathrm{C}$ in whorls three and four. The overlapping combination of these activities in different whorls provides an 'address' that is sufficient to specify a particular organ identity in each whorl (Fig. 1). Mutations in the Arabidopsis APETALAI (API) and APETALA2 (AP2) genes both disrupt the specification of sepals and petals, and so are required for A activity (Bowman, et al., 1989; Irish and Sussex, 1990). Mutations in the APETALA3 (AP3) and PISTILLATA (PI) genes cause homeotic transformations of the petals and stamens and so are required for B activity, while the AGAMOUS (AG) gene product is required for the specification of stamens and carpels and thus confers $\mathrm{C}$ activity (Bowman, et al., 1989).

All of these genes have now been cloned, and $A P 1, A P 3, P I$ and $A G$ encode members of the MADS box family of transcription factors, while $A P 2$ encodes a product with similarity to the EREBP family of transcriptional regulators (Yanofsky, et al., 1990; Jack, et al., 1992; Mandel, et al., 1992; Goto and Meyerowitz, 1994; Jofuku, et al., 1994; Weigel, 1995). More recently, three MADS box genes with redundant functions (SEPALLATA1, 2, and 3) have been isolated and have been

Received for publication 16 Oct. 2001. Accepted for publication 4 Feb. 2003. Work on floral development in my laboratory is supported by grants from the National Science Foundation and U.S. Department of Agriculture shown to also be required for specifying petal, stamen, and carpel identities, adding another layer of complexity to the ABC model (Pelaz, et al., 2000) (Fig. 1). These MADS-box gene products form ternary and quaternary complexes which could explain, at the molecular level, how these transcription factors coordinate their functions (Honma and Goto, 2001; Pelaz, et al., 2001). The expression patterns of all of these genes, with the exception of $A P 2$ which is expressed ubiquitously throughout the flower, largely corresponds to their domains of action as postulated in the $\mathrm{ABC}$ model.

\section{The roles of $A P 3$ and $P I$ in Arabidopsis}

As demonstrated by their mutant phenotypes, $A P 3$ and $P I$ are both necessary to specify petal and stamen identities. Ectopic expression studies show that $A P 3$ and $P I$ together are also sufficient to confer petal and stamen identities. By ectopically expressing $A P 3$ and $P I$ together in the first whorl, for instance, almost completely normal petals arise in place of sepals (Krizek and Meyerowitz, 1996) (R.S. Lamb and V.F.I, unpublished). Ectopic expression of AP3 or PI alone, however, is insufficient to cause a homeotic transformation. The requirement for both $A P 3$ and $P I$ gene products to elicit a homeotic conversion can be explained by the observation that $A P 3$ and $P I$ proteins must form a heterodimer in order to bind to DNA and transcriptionally regulate downstream target genes (Riechmann, et al., 1996; Hill, et al., 1998).

$A P 3$ and $P I$ are initially expressed at an early stage in floral development, when the sepal primordia first appear. Both $A P 3$ and $P I$ are expressed ubiquitously in the petal and stamen primordia once they arise, and this pattern of expression persists throughout the development of these organ types (Jack, et al., 1992; Goto and Meyerowitz, 1994). In order to test whether $A P 3$ expression is actually required in all of these developing petal and stamen cells for normal development to ensue, mosaic analyses have been carried out. By generating flowers which contain both $A P 3$ wild type and $A P 3$ mutant cell layers, the requirements for $A P 3$ expression in different tissues can be examined. Such mosaic flowers can be generated in Arabidopsis using a heterologous maize Ds transposable element that has been engineered to express $A P 3$; transposase-induced excision of this construct produces a clonal patch of AP3 mutant cells in an otherwise wild type background (Jenik and Irish, 2001). A similar strategy can be used to generate a clone
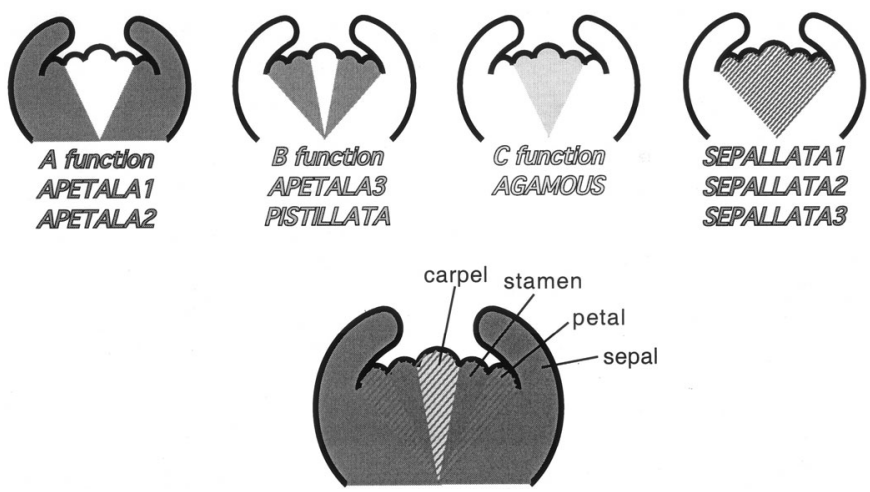

Fig. 1. The ABC model. The overlap of three functions, A,B, and C, in differ ent regions of the floral meristem (here shown after sepal primordia have arisen) is sufficient to specify different floral organ identities (Coen and Meyerowitz, 1991). In Arabidopsis, these functions are encoded by the APETALA1, APETALA2, APETALA3, PISTILLATA, and AGAMOUS genes. In addition, the functions of the SEPALLATA1, 2, and 3 genes also are required for specification of the different floral organ identities in Arabidopsis (Honma and Goto, 2001). 
of cells in which $A P 3$ expression has been reconstituted in otherwise AP3 mutant tissue.

This approach has been used to investigate the role of $A P 3$ in petal and stamen development, and has demonstrated that $A P 3$ has both autonomous and non-autonomous functions in the development of these organ types (Jenik and Irish, 2001). Arabidopsis petals consist of two clonally derived tissue types: the epidermis which is a single cell layer thick sheet of conical cells overlying the internal mesophyll, which in mature petals contains leukoplasts giving the petal blade a white color (Pyke and Page, 1998; Jenik and Irish, 2000). When expression of $A P 3$ is limited to the epidermis, petal-type epidemal cell type differentiation occurs and the organs have green sepal-like mesophyll, indicating that $A P 3$ acts autonomously to confer cell-type specific differentiation. However, AP3 expression that is limited to just the epidermis is sufficient to confer a normal petal shape, indicating that $A P 3$ acts in a non-cell-autonomous fashion to confer the petal-type growth parameters on the underlying mesophyll. Furthermore, expression of $A P 3$ in just the mesophyll resulted in leukoplast differentiation, but abnormal, sepal-shaped organs, underscoring the requirement for $A P 3$ in the epidermis to confer a normal spatulate petal shape (Jenik and Irish, 2001). Mosaic analyses in the stamens have also been carried out, and demonstrate that $A P 3$ also regulates many aspects of stamen differentiation in a noncell-autonomous fashion. The response of $A P 3$ mutant cells to the action of $A P 3$ in neighboring cells demonstrates that $A P 3$ is involved in cell-cell signaling, presumably by regulating the expression of downstream target genes required for cell-cell signaling, since the $A P 3$ protein itself does not move between cells (Jenik and Irish, 2001). Together, these results demonstrate that $A P 3$ is required in virtually all cell types of the developing petals and stamens to regulate the cell-cell interactions that are necessary for normal development to ensue.

\section{To what extent are the functions of $A P 3$ and $P I$ conserved in other angiosperm species?}

Homologs of $A P 3$ and $P I$ have now been cloned from a variety of other plant species (reviewed in Irish and Kramer, 1998; Theissen, et al., 2000). The Arabidopsis AP3 and PI genes are more closely related to each other than to any other MADS box genes, suggesting these genes arose from the duplication of an ancestral gene. Phylogenetic analyses of the $A P 3$ and $P I$-like genes support this idea and place this duplication event as occurring prior to the origin of the angiosperms (Purugganan, et al., 1995; Purugganan, 1997; Kramer, et al., 1998; Theissen, et al., 2000). Several gymnosperm genes with similarities to $A P 3$ and $P I$ lineage genes have also been identified, but it remains unclear as to whether they group with $A P 3, P I$, or both, or if they instead represent a basal lineage (Mouradov, et al., 1999; Sundstrom, et al., 1999). Thus, the duplication event which produced the $A P 3$ and PI gene lineages appears to have occurred at least 200 million years ago, and perhaps much earlier.

Several phylogenetic analyses of the angiosperms have recently been carried out using different datasets, and these studies have, for the first time, converged upon a consistent relationship among different angiosperm species (Mathews and Donoghue, 1999; Soltis, et al., 1999; Barkman, et al., 2000; Chaw, et al., 2000). These analyses demonstrate that the eudicots and monocots each form a well-supported monophyletic clade, and also indicate the relationships among basal angiosperms (Fig. 2). Within the eudicots, a clade containing the rosids, asterids and caryophyllids, can be defined and contains the vast majority of eudicot species. Species within this 'core eudicot' clade display much less variability in their floral architecture than do other angiosperm species. These core eudicots are typified by having whorled, as opposed to spiral, floral phyllotaxy, as well as having a fixed number of floral organs for any given species (Magallon, et al., 1999). Furthermore, morphological analyses suggest that petals have arisen independently at the base of the core eudicots. (Takhtajan, 1991). This fixation of core eudicot floral architecture is thought to reflect a 'key innovation', or an indispensible stabilization of structural elements, in flower evolution (Endress, 2001).

Phylogenetic analyses of $A P 3$-like sequences indicate that a major duplication event has also taken place during the evolution of the an-

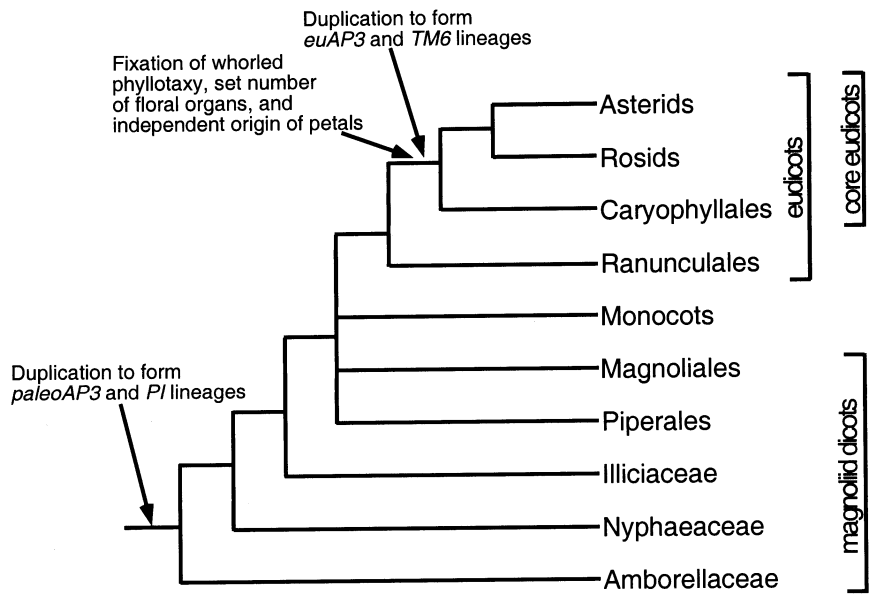

Fig. 2. Major events in core eudicot floral evolution. A simplified version of the current angiosperm phylogeny is shown (Soltis, et al., 1999). The postulated occurrence of major duplication events in the $A P 3$ and $P I$ lineages, and key morphological innovations in core eudicot floral architecture are indicated (Kramer and Irish, 1999).

giosperms, concomitant with the diversification of the core eudicots (Kramer, et al., 1998). This duplication event resulted in two lineages; the euAP3 lineage, which includes Arabidopsis AP3, and the TM6 lineage, named after the founding member of this family of genes in tomato (Fig. 2). Outside of the core eudicots, AP3-like genes have sequence motifs that are more similar to their TM6 counterparts than to the $e u A P 3$ genes, suggesting that the duplication event that produced the $e u A P 3$ genes may have allowed for the diversification of function in this lineage. In addition, preliminary evidence indicates that a major duplication event in the $A P I$ gene lineage has also occurred at the base of the core eudicots (A. Litt and V.F.I. unpublished). Together, these observations suggest that significant changes in the underlying genetic programs controlling floral patterning may have occurred concomitant with the origin of the core eudicots.

Does the correlation between the key innovation in core eudicot evolution and MADS box gene duplication events reflect an underlying change in the roles of the MADS box gene products? One way to examine this possibility is by determining if changes in the expression, and presumably function, of these MADS box genes parallels the changes in floral morphological evolution. As discussed above, the Arabidopsis AP3 and PI genes are expressed in all cells of the developing petal and stamen primordia throughout their development, and, as has been shown for $A P 3$, this pattern of expression is critical for normal organogenesis to ensue. In the other core eudicots that have been examined, the expression patterns of $e u A P 3$ and $P I$ lineage genes are also ubiquitous throughout the developing petal and stamen primordia (reviewed in Irish and Kramer, 1998). In contrast, the expression of the paleoAP3 and $P I$-like genes in lower eudicots is largely limited to the developing stamens, while expression in the developing petals is either transient, spatially restricted, or absent (Kramer and Irish, 1999). This lack of constant and uniform expression in the petals implies that the paleoAP3 and PI-like genes in the lower eudicots do not function in petal development in the same way as their core eudicot counterparts. The expression of $A P 3$ - and $P I$-lineage genes in stamen primordia appears to be constant in all angiosperm species examined (reviewed in Irish and Kramer, 1998; Irish, 2000). Furthermore, a few AP3 and $P I$-like genes have now been identified in gymnosperms and have also been found to be expressed in developing male strobili (Mouradov, et al., 1999; Sundstrom, et al., 1999; Winter, et al., 1999).

Together, these observations support a model in which the ancestral function of the AP3 and PI lineage genes was in specifying male reproductive development (Irish, 2000). In the core eudicots, these gene functions were presumably recruited for an additional task, that of also specifying petal organ identity. In the lower eudicots, in contrast, it appears the paleoAP3 and PI genes do not function in the same manner as their core eudicot counterparts in specifying petal identity, and in fact may have no function in petal development. This model has a strong prediction: loss of function of the paleoAP 3 and 
PI genes in a lower eudicot should only disrupt the specification of stamen identity, and have little or no affect on petal identity. Such loss of paleoAP3 and $P I$ gene function can be engineered in a wide variety of species using transgenic approaches. By dissecting the roles of $A P 3$ and $P I$ homologs in lower eudicot species, the roles of these genes in various developmental processes can be characterized and can be used to elucidate how changes in developmental programs may have led to the evolution of new floral forms.

\section{Prospects}

The analyses of the roles of AP3 and PI homologs in other angiosperm species points out the limitations in extrapolating functions from one example to phylogenetically diverse species. Future work aimed at genetic engineering of flower form or reproductive capacities in non-model plant species will require a more sophisticated understanding of how these floral developmental pathways have been modulated over evolutionary time. In turn, this information will be crucial in developing targeted strategies to specifically manipulate developmental pathways of interest.

\section{Literature Cited}

Barkman, T.J., G. Chenery, J.R. McNeal, J. Lyons-Weiler, and E.J. Ellisens, et al. 2000. Independent and combined analyses of sequences from all three genomic compartments converge on the root of flowering plant phylogeny. Proc. Nat. Acad. Sci. 97:13166-71

Bowman, J.L., D.R. Smyth, and E.M, Meyerowitz. 1989. Genes directing flower development in Arabidopsis. Plant Cell. 1:37-52

Chaw, S.M., C.L. Parkinson, Y. Cheng, T.M. Vincent, and J.D. Palmer. 2000. Seed plant phylogeny inferred from all three plant genomes: Monophyly of extant gymnosperms and origin of Gnetales from conifers. Proc. Nat. Acad. Sci. 97:4086-91

Coen,E.S. and E.M. Meyerowitz. 1991. The war of the whorls: Genetic interactions controlling flower development. Nature. 353:31-7.

Endress, P.K. 2001. Origins of flower morphology. J. Exp. Zool. 291: $105-15$.

Goto, K. and E.M. Meyerowitz. 1994. Function and regulation of the Arabidopsis floral homeotic gene PISTILLATA. Genes and Dev. 8:1548-60

Hill, T.A., C.D. Day, S.C.Zondlo, A.G. Thackeray, and V.F. Irish. 1998. Discrete spatial and temporal cis-acting elements regulate transcription of the Arabidopsis floral homeotic gene APETALA3. Development 125:1711-21.

Honma, T. and K. Goto. 2001. Complexes of MADS-box proteins are sufficient to convert leaves into floral organs. Nature. 409:469-71.

Irish, V.F. 2000. Variations on a theme: Flower development and evolution. Genome Biol. 1:1015.1-.4

Irish, V.F. and E.M. Kramer. 1998. Genetic and molecular analysis of Angiosperm flower development. Adv. Bot. Res. 28:197-230.

Irish, V.F. and I.M. Sussex. 1990. Function of the apetala-1 gene during Arabidopsis floral development. The Plant Cell. 2:741-53.

Jack, T., L.L. Brockman, and E.M. Meyerowitz. 1992. The homeotic gene APETALA3 of Arabidopsis thaliana encodes a MADS box and is expressed in petals and stamens. Cell. 68:683-97.

Jenik, P.D. and V.F. Irish. 2000. Regulation of cell proliferation patterns by homeotic genes during Arabidopsis floral development. Development 127:1267-76

Jenik, P.D. and V.F. Irish. 2001. The Arabidopsis floral homeotic gene
APETALA3 differentially regulates intercellular signaling required for petal and stamen development. Development. 128:13-23.

Jofuku, K.D., B.G.W. den Boer, M. Van Montague, and J.K. Okamuro. 1994 Control of Arabidopsis flower and seed development by the homeotic gene APETALA2. Plant Cell. 6:1211-25.

Kramer,E.M., R.L. Dorit, and V.F. Irish. 1998. Molecular evolution of petal and stamen development: Gene duplication and divergence within the APETALA3 and PISTILLATA MADS-box gene lineages. Genetics. 149:765-83.

Kramer, E.M. and V.F. Irish. 1999. Evolution of genetic mechanisms controlling petal development. Nature. 399:144-8.

Krizek, B.A. and E.M. Meyerowitz. 1996. The Arabidopsis homeotic genes APETALA3 and PISTILLATA are sufficient to provide the B class organ identity function. Development. 122:11-22.

Magallon, S., P.R. Crane, and P.S. Herendeen. 1999. Phylogenetic pattern, diversity, and diversification of Eudicots. Ann. Mo. Bot. Gard. 86:297-372.

Mandel, M.A., C. Gustafson-Brown, B. Savidge, and M.F. Yanofsky. 1992. Molecular characterization of the Arabidopsis floral homeotic gene APETALA1. Nature. 360:273-7.

Mathews, S. and M.J. Donoghue. 1999. The root of angiosperm phylogeny inferred from duplicate phytochrome genes. Science. 286:947-50.

Mouradov, A., B. Hamdorf, R.D. Teasdale, J. Kim, K.-U. Winter, and G. Theissen. 1999. A DEF/GLO like MADS box gene from a gymnosperm: Pinus radiata contains an ortholog of angiosperm $\mathrm{B}$ class floral homeotic genes. Devel. Genetics. 25:245-52.

Pelaz, S., G.S. Ditta, E. Baumann, E. Wisman, and M.F. Yanofsky. 2000. B and $\mathrm{C}$ floral organ identity functions require SEPALLATA MADS-box genes. Nature. 405:200-3.

Pelaz, S., R. Tapia-Lopez, E.R. Alvarez-Buylla, and M.F. Yanofsky. 2001. Conversion of leaves into petals in Arabidopsis. Curr. Biol. 11:182-4.

Purugganan, M.D. 1997. The MADS-box floral homeotic gene lineages predate the origin of seed plants: phylogenetic and molecular clock estimates. J. Mol. Evol. 45:392-6.

Purugganan, M.D., S.D. Rounsley, R.J. Schmidt, and M.F. Yanofsky. 1995. Molecular evolution of flower development: Diversification of the plant MADS box regulatory gene family. Genetics. 140:345-56.

Pyke, K.A. and A.M. Page. 1998. Plastid ontogeny during petal development in Arabidopsis. Plant Physiol. 116: 797-803.

Riechmann, J.L., M. Wang, and E.M. Meyerowitz. 1996. DNA-binding properties of Arabidopsis MADS domain homeotic proteins APETALA1, APETALA3, PISTILLATA and AGAMOUS. Nucl. Acids Res. 24: 3134-41.

Soltis, P.S., Soltis, D.E., Chase, M.W. 1999. Angiosperm phylogeny inferred from multiple genes as a tool for comparative biology. Nature. 402:402-4.

Sundstrom, J., A. Carlsbecker, M. Svenson, M.E. Svensson, and P. Engstrom 1999. MADS box genes active in developing pollen cones of Norway Spruce are homologous to the $\mathrm{B}$ class floral homeotic genes in angiosperms. Devel. Genetics. 25:253-66.

Takhtajan, A. 1991. Evolutionary trends in flowering plants. Columbia Univ. Press. New York.

Theissen, G., A. Becker, A. Di Rosa, A. Kanno, and J.T. Kim, et al. 2000. A short history of MADS-box genes in plants. Plant Mol. Biol. 42:115-49.

Weigel, D. 1995. The APETALA2 domain is related to a novel type of DNA binding domain. Plant Cell 7:388-9.

Winter, K.-U., Becker, A., Munster, T., Kim, J.T., Saedler, H., Theissen, G. 1999. MADS box genes reveal that gnetophytes are more closely related to conifers than to flowering plants. Proc. Nat. Acad. Sci. 96:7342-7.

Yanofsky, M.F., Ma, H., Bowman, J.L., Drews, G.N., Feldmann, K.A., Meyerowitz, E.M. 1990. The protein encoded by the Arabidopsis homeotic gene agamous resembles transcription factors. Nature. 346:35-9. 